\title{
Cartographie des biocénoses marines de Guadeloupe à partir de données SPOT (récifs coralliens, phanérogames marines, mangroves)
}

\author{
Sylvain CHAUVAUD ${ }^{\mathrm{a} *}$, Claude BOUCHON ${ }^{\mathrm{b}}$, Roger MANIÈRE ${ }^{\mathrm{c}}$ \\ ${ }^{a}$ Université de Bretagne Occidentale, Bioflux, IUEM, technopôle Brest Iroise, 29280 Plouzané, France \\ ${ }^{\mathrm{b}}$ Laboratoire de biologie animale, université Antilles-Guyane, BP 592, 97159 Pointe-à-Pitre, Guadeloupe, France \\ c URA 1152-CNRS, faculté de Saint-Jérôme, université Aix-Marseille 3, 13397 Marseille, France
}

Reçu le 18 février 1999 ; révisé le 23 décembre 1999 ; accepté le 7 janvier 2000

\begin{abstract}
Thematic mapping of tropical marine communities (coral reefs, seagrass beds and mangroves) using SPOT data in Guadeloupe Island. In the framework of a comprehensive study of the coastal marine ecosystems of Guadeloupe Island (French West Indies), a remote sensing survey of marine communities was conducted in the Bay of the Grand Cul-de-Sac Marin. Pictures of SPOT satellite were used. At first sight, XS data from SPOT did not seem to be well adapted to extract information from deepest underwater areas. In the present study, a method for analysis of XS data was developed, which increases tenuous spectral differences associated to very low intensity signals. This technique led to a better distinction of objects, even the deepest ones. Reference surfaces were then identified in the field and a reliable supervised classification was conducted, which reflected the high diversity of ecological themes (up to 32) of the area to $20 \mathrm{~m}$ depth. A total of 23700 ha of biota was clearly identified. Mangrove covered up to $2700 \mathrm{ha}$. Submerged areas were constituted of 15700 ha of soft bottoms and 3800 ha of hard substrate bottoms. On the coral reefs, flourishing coral communities accounted only for 113 ha $(11.2 \%)$. This result enhances the alarming situation of these communities in Guadeloupe. Finally, seagrass beds took up to 8200 ha (i.e. $42 \%$ of the total sublittoral area, $58 \%$ of soft bottom substrates). This ecosystem revealed to be of primary importance within the studied area and particular attention was paid to seagrass beds of Thalassia testudinum. Twelve themes were retained to describe them, based on the coverage rate of the substratum by the plants and the type of the sediment colonised. The final document expresses well the high complexity of the studied area. This map constitutes a reference of the present status of the coastal benthic communities of the 'Grand Cul-de-Sac Marin' and can be useful for scientists and the administrative managers of that area. (C) 2001 Ifremer/CNRS/IRD/Éditions scientifiques et médicales Elsevier SAS
\end{abstract}

Résumé - Dans le cadre d'une étude des écosystèmes marins côtiers de l'île de la Guadeloupe, un inventaire des biocénoses marines de la baie du Grand Cul-de-Sac Marin a été réalisé. Dans les Antilles, ces biocénoses sont souvent intriquées de façon complexe, ce qui rend leur cartographie difficile. Les techniques de télédétection spatiale et aéroportée peuvent apporter des solutions à ce problème. Dans le présent travail, une méthode d'analyse des données XS du satellite SPOT a été mise au point pour améliorer nettement la discrimination des objets, même profonds, en amplifiant des différences spectrales minimes associées à un signal de très faible intensité. Des surfaces de référence sont délimitées en vue de construire une classification supervisée ; 32 thèmes sont identifiés sur une superficie de 23700 ha, jusqu'à l'isobathe $20 \mathrm{~m}$. La mangrove occupe 2700 ha et les surfaces immergées incluent 3800 ha de fonds durs et

*Correspondance et tirés à part.

Adresse e-mail : sylvain.chauvaud@ifremer.fr (S. CHAUVAUD).

(C) 2001 Ifremer/CNRS/IRD/Éditions scientifiques et médicales Elsevier SAS. Tous droits réservés 
15700 ha de fonds meubles. Sur les récifs, les communautés coralliennes florissantes ne représentent que 113 ha, soit $11,2 \%$ de la surface des récifs cartographiés. Ce résultat met en évidence l'état alarmant des récifs coralliens en Guadeloupe. Sur les fonds sédimentaires, une attention spéciale est accordée aux herbiers de phanérogames marines qui constituent un faciès particulier occupant 8200 ha, soit 42\% des biocénoses infralittorales cartographiées et $58 \%$ des fonds meubles. Leur description fait l'objet de 12 thèmes, différenciés sur la base du taux de recouvrement des fonds par les phanérogames et du type de sédiment colonisé par ces plantes. La méthode d'inventaire cartographique (analyse de données SPOT) révèle la grande complexité de la zone d'étude. La carte produite constitue un outil de référence à la disposition des scientifiques et des gestionnaires de cet espace. () 2001 Ifremer/CNRS/IRD/Éditions scientifiques et médicales Elsevier SAS

\section{remote sensing / mapping / coral reef / seagrass / mangrove}

\section{télédétection / cartographie / récif corallien / phanérogame marine / mangrove}

\section{INTRODUCTION}

La zone d'étude est située au nord de l'île de la Guadeloupe dans la baie du Grand Cul-de-Sac Marin (figure 1), l'un des lagons les plus étendus des Petites Antilles. Cette zone est classée réserve «Man and Biosphere » (MAB) par l'Unesco et une partie du lagon et du récif constitue la réserve naturelle du Grand Cul-de-Sac Marin, gérée par le Parc National de la Guadeloupe.

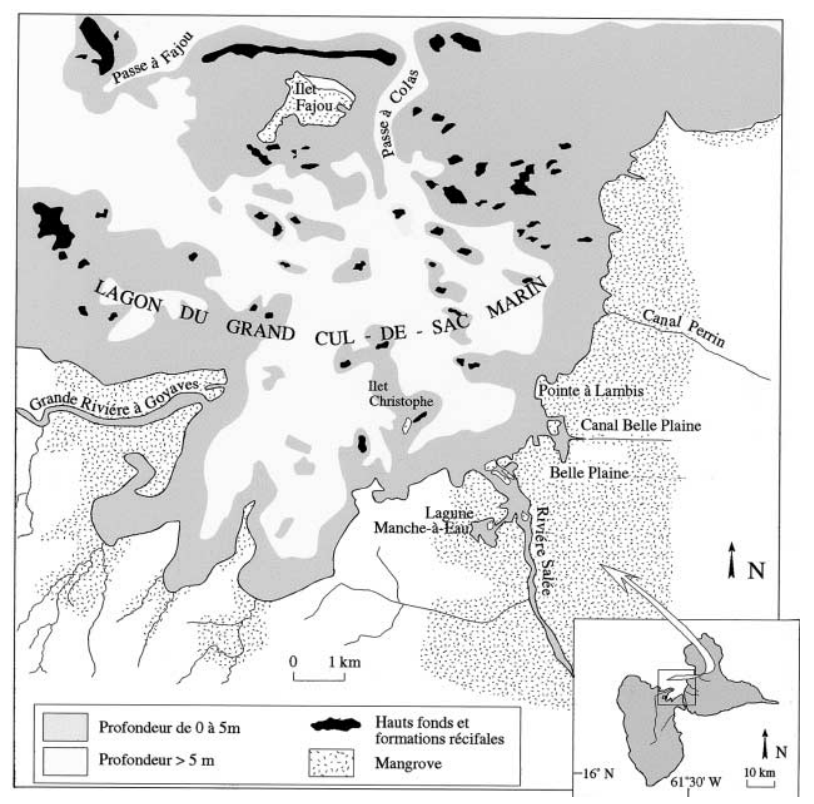

Figure 1. Île de la Guadeloupe. Localisation du Grand Cul-de-Sac Marin.

Figure 1. Guadeloupe Island. Localization of the Grand Cul-de-Sac Marin.
Dans la région Caraïbe, la frange littorale est caractérisée par la présence de communautés benthiques particulières comme les biocénoses récifales, sur les substrats durs, et les herbiers de phanérogames marines ou les mangroves, sur les fonds meubles. Dans les Antilles, ces communautés sont souvent intriquées de façon complexe, ce qui rend leur cartographie difficile. Les techniques de télédétection spatiale ou aéroportée sont susceptibles d'apporter des réponses aux problèmes d'inventaire de ces milieux (Bina et al., 1978 ; Claasen et Pirazzoli, 1984 ; Jupp, 1983 ; Jupp et al., 1981a et b, 1985 , Mayo et Jupp, 1982).

Dans le cadre d'une étude générale des écosystèmes marins côtiers des Antilles françaises, développée par l'université des Antilles et de la Guyane, ce travail s'inscrit dans un programme de recherche sur les herbiers de phanérogames marines, leur distribution, leur importance en tant que biomasse végétale et leur production primaire (Chauvaud, 1997). Les résultats sont intégrés au système d'information géographique (SIG) du Parc National de la Guadeloupe, afin de constituer une information disponible pour des plans d'aménagement et de protection de l'environnement du Grand Cul-de-Sac Marin.

Déjà utilisées par différents auteurs pour réaliser avec succès des cartographies de zones côtières tropicales (Belsher et al., 1990 ; Bour et al., 1986 ; Courboulès et al., 1987, 1988 ; Loubersac et Populus, 1986), les données du satellite SPOT ont été retenues ici. La méthode d'analyse est décrite et l'intérêt d'une carte thématique à haute résolution est discuté. 


\section{MATÉRIEL ET MÉTHODES}

\subsection{Données disponibles}

Trois types de données ont été utilisés : a) une scène SPOT acquise en modes panchromatique et multispectral (respectivement de résolution $10 \mathrm{~m}$ et $20 \mathrm{~m}$ ), le 01/02/ 1994 à 14 h 48 T.U. (scène 667-318/0) ; b) deux cartes marines du Service hydrographique et océanographique de la marine, SHOM no 7302 et $n^{\circ} 7324$, couvrant la zone étudiée; c) des relevés de terrain géoréférencés, réalisés au cours de l'année 1994, décrivant les substrats et leur couverture biologique.

\subsection{Redressement et masquage des zones émergées}

Un redressement automatique (niveau 2B) de la scène SPOT a été effectué par la société SPOT Image. La localisation du canal XS3 dans le proche infrarouge permet de masquer les zones émergées et les nuages. Les cartes marines ont été numérisées et recalées géographiquement afin d'être superposables à la scène SPOT.

\subsection{Création de néo-canaux}

Le canal XS3 $(0,79-0,89 \mu \mathrm{m})$ de SPOT fournit essentiellement des informations sur les formations émergées (palétuviers des mangroves et marais côtiers). Les canaux $X S 1 \quad(0,50-0,59 \mu \mathrm{m})$ et $X S 2(0,61-0,69 \mu \mathrm{m})$ sont plus utiles pour l'étude des communautés immergées (figure 2). Cependant, la forte corrélation liant ces deux derniers canaux n'est pas favorable à l'analyse des données spectrales (Belsher et al., 1990; Bour et al., 1986 ; Courboulès et al., 1987, 1988 ; Loubersac et Populus, 1986 ; Thomassin et al., 1985). Les objets à cartographier présentent une faible luminance et une similitude importante de leurs signatures spectrales qui les rendent difficiles à discriminer. Pour remédier à ces problèmes, plusieurs canaux artificiels appelés «néo-canaux » ont été créés.

Le premier néo-canal (Can1) est construit de façon à conserver la plus grande part de la variance contenue dans les canaux XS de SPOT (figure 2). Une analyse en composantes principales $(\mathrm{ACP})$ est réalisée sur le fichier issu de la composition trichrome des canaux XS1, XS2 et XS3, après application du masque. Les coordonnées des points sur chacun des trois axes factoriels $(\mathrm{Cl}, \mathrm{C} 2, \mathrm{C} 3)$ permettent de construire le premier néo-canal : l'information contenue dans ces fichiers est synthétisée en additionnant ces valeurs, pondérées par le poids relatif de chacun des axes (pourcentage de variance expliquée par chaque valeur propre) (figure 2). Dans un travail similaire, Manière et al. (1991) n'avaient conservé que le premier axe. Dans la présente étude, il est apparu nécessaire de retenir les trois axes, dans la mesure où les axes 2 et 3 apportent l'essentiel de l'information relative aux biotopes situés à faible profondeur :

$$
\text { Canl }=(0,747 \times C 1)+(0,178 \times C 2)+(0,074 \times C 3)
$$

Pour le deuxième néo-canal (Can2), la combinaison proposée par Courboulès et al. (1992) se révèle pertinente pour séparer les différents types d'herbiers et discriminer les objets les plus profonds (figure 2) :

$$
\text { Can } 2=(X S 1-X S 2)^{2} \times X S 2^{-1}
$$

La combinaison retenue pour élaborer le troisième néocanal (Can3) permet d'accentuer les différences de signature spectrale entre les différents types de fonds de couleurs sombres, particulièrement dans les zones proches de la mangrove (figure 2) :

$$
\text { Can3 }=\exp \left(X S 1 \times X S 2^{-1}\right)
$$

De nombreuses autres combinaisons, issues ou non de la littérature, ont été testées.

Le tableau Ia présente les coefficients de corrélations entre les trois canaux XS, après masquage des zones terrestres et des nuages. Ces coefficients sont très élevés, particulièrement entre XSI et XS2. Le tableau Ib montre que les néo-canaux, Can1, Can2 et Can3, sont moins corrélés entre eux que les canaux bruts, ce qui favorise la discrimination des pixels.

La figure $3 a$ représente une composition trichrome de la région centrale du lagon, réalisée à partir des données XS de SPOT après masquage des zones terrestres et ennuagées, puis rehaussement du contraste. Son examen montre que les objets profonds y sont peu discernables. La figure $3 b$ correspond à une trichromie de cette même région, réalisée à partir des néo-canaux et suivie par un rehaussement du contraste. Sa comparaison avec la figure précédente révèle que la méthode mise au point améliore nettement la discrimination spectrale des objets. 

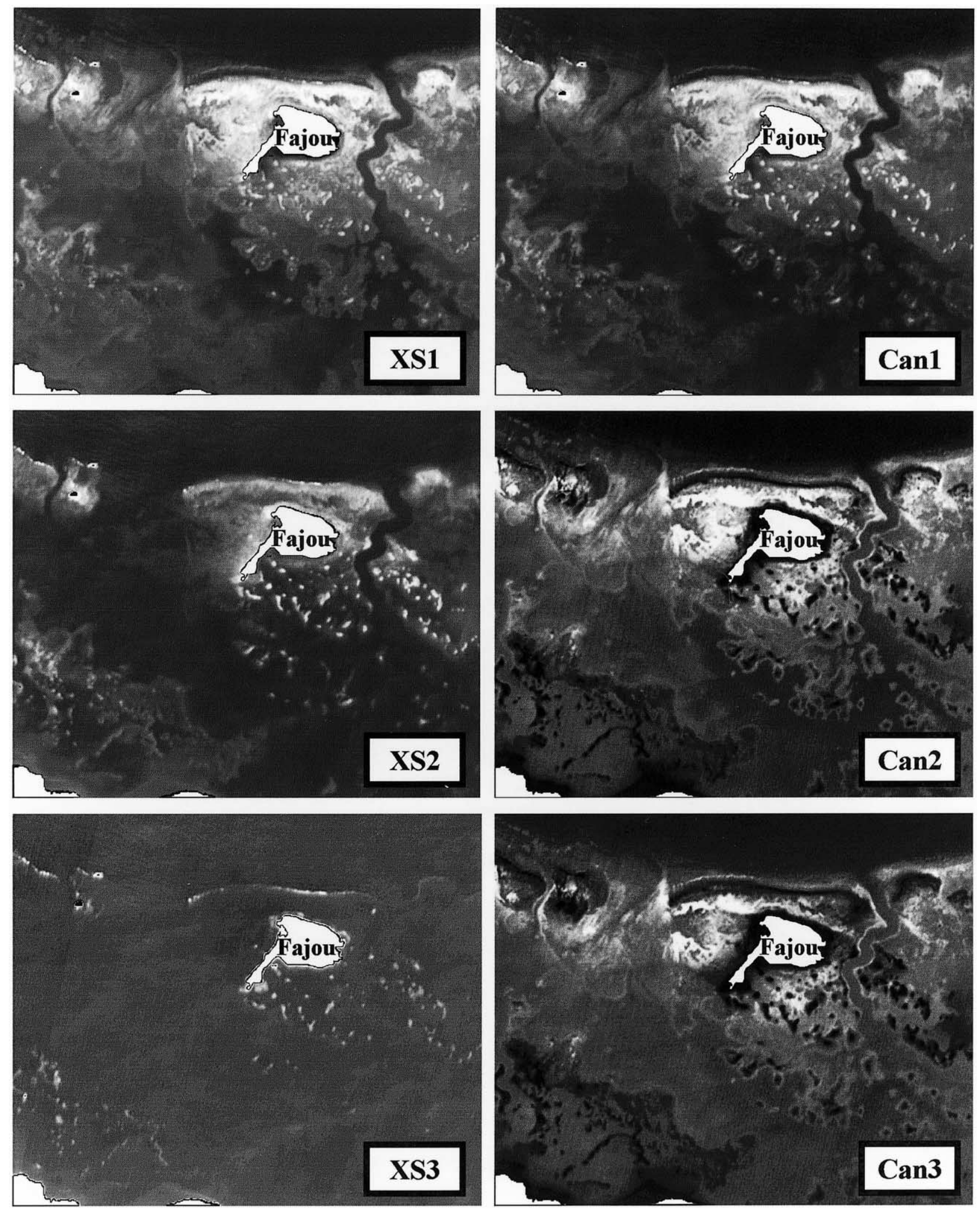
Tableau I. (a) Matrice de corrélation des canaux XS après masquage des parties émergées. (b) Matrice de corrélation des canaux Can1, Can2 et Can3.

Table I. a) Correlation matrix of XS channels after terrestrial areas were masked. b) Correlation matrix of Can1, Can2 et Can3 channels.

\begin{tabular}{|c|c|c|c|c|c|c|c|}
\hline (a) & XS1 & XS2 & XS3 & (b) & Can1 & Can2 & Can3 \\
\hline XS1 & 1 & & & Can1 & 1 & & \\
\hline XS2 & 0,97 & 1 & & Can2 & 0,83 & 1 & \\
\hline XS3 & 0,84 & 0,91 & 1 & Can3 & 0,82 & 0,64 & 1 \\
\hline
\end{tabular}

\section{4. Élaboration de masques}

Du fait de l'absorption de la lumière par l'eau, la profondeur et la turbidité interfèrent dans l'identification et la classification des biotopes étudiés. Dans le lagon du Grand Cul-de-Sac Marin, la présence d'un gradient de turbidité, d'origine terrigène, décroissant de la côte vers le large, ne permet pas d'effectuer un premier découpage de l'image à étudier en élaborant des masques uniquement basés sur un critère bathymétrique extrait de la carte marine. En pratique, après la première classification non supervisée, le fichier a été scindé en deux sous-unités correspondant à deux grandes classes de pixels, l'une pour les zones de faible profondeur et l'autre pour les eaux plus profondes et les secteurs très turbides. Les trichromies «optimisées » construites pour chacune de ces zones ont servi de fichiers de données pour les traitements ultérieurs.

\subsection{Délimitation des parcelles tests}

Les deux fichiers correspondant aux compositions trichromes obtenues par la méthode citée plus haut ont été analysés. Une classification non supervisée définit un certain nombre de classes de pixels décrivant l'ensemble de l'espace spectral et permettant de visualiser les principaux objets identifiés sur le terrain lors d'une mission exploratoire. Une vingtaine de classes est retenue pour chacune des deux compositions. Pour définir des surfaces de référence (parcelles tests), certaines contraintes doivent être prises en compte.
Justice et Townshed (in Bainbridge et Reichelt, 1988) ont défini la MDU (Minimum Discernible Unit) comme suit :

$$
\mathrm{A}=[P(1+2 L)]^{2}
$$

où $A=$ aire de la MDU (en $\left.\mathrm{m}^{2}\right)$

$P=$ dimensions du pixel $(20 \mathrm{~m})$

$L=$ précision dans la localisation du pixel sur l'image (1 pixel)

Pour les données multispectrales de SPOT, cette formule produit une MDU de $60 \mathrm{~m} \times 60 \mathrm{~m}$. En pratique, cette contrainte conduit à délimiter des parcelles mesurant au moins trois pixels de côté.

Dans une famille de parcelles tests, le spectre de chaque canal doit approcher une distribution gaussienne et, pour être représentative, chaque famille de parcelles doit être constituée d'au moins 50 pixels (Fralit, 1981). Ces contraintes font que des biocénoses très peu représentées ne peuvent être prises en compte.

Les parcelles tests sont construites en utilisant les résultats des classifications non supervisées. Une famille de parcelles ne contient qu'une seule classe de pixels et doit présenter un spectre unimodal dans chacun des canaux. Le résultat des classifications est affiché sur l'écran de l'ordinateur et, pour chaque classe, une ou plusieurs parcelles sont délimitées et saisies. Par cette technique 250 parcelles sont retenues pour décrire la totalité de l'espace spectral étudié.

\subsection{Vérité de terrain}

Le travail de « vérité terrain » a été effectué dans les mois qui ont suivi la réalisation de la scène SPOT et 250 parcelles tests sont reportées sur les cartes marines numérisées. Les coordonnées du centre des parcelles ont été relevées sur la carte. Un système de navigation GPS (Global Positioning System) non différentiel a permis de se positionner sur le terrain à l'intérieur de chaque parcelle. L'erreur moyenne de position GPS étant de l'ordre de $30 \mathrm{~m}$ et les parcelles ayant toutes plus de $60 \mathrm{~m}$

Figure 2. Vue du centre du lagon (ilet Fajou) dans les canaux XS1, XS2 et XS3 après masquage des zones terrestres et rehaussement du contraste et dans les canaux Can1, Can2 et Can3.

Figure 2. View of the central part of lagoon (ilet Fajou) with XS1, XS2 and XS3 channels after terrestrial areas were masked and with Can1, Can2 and Can3 channels. 

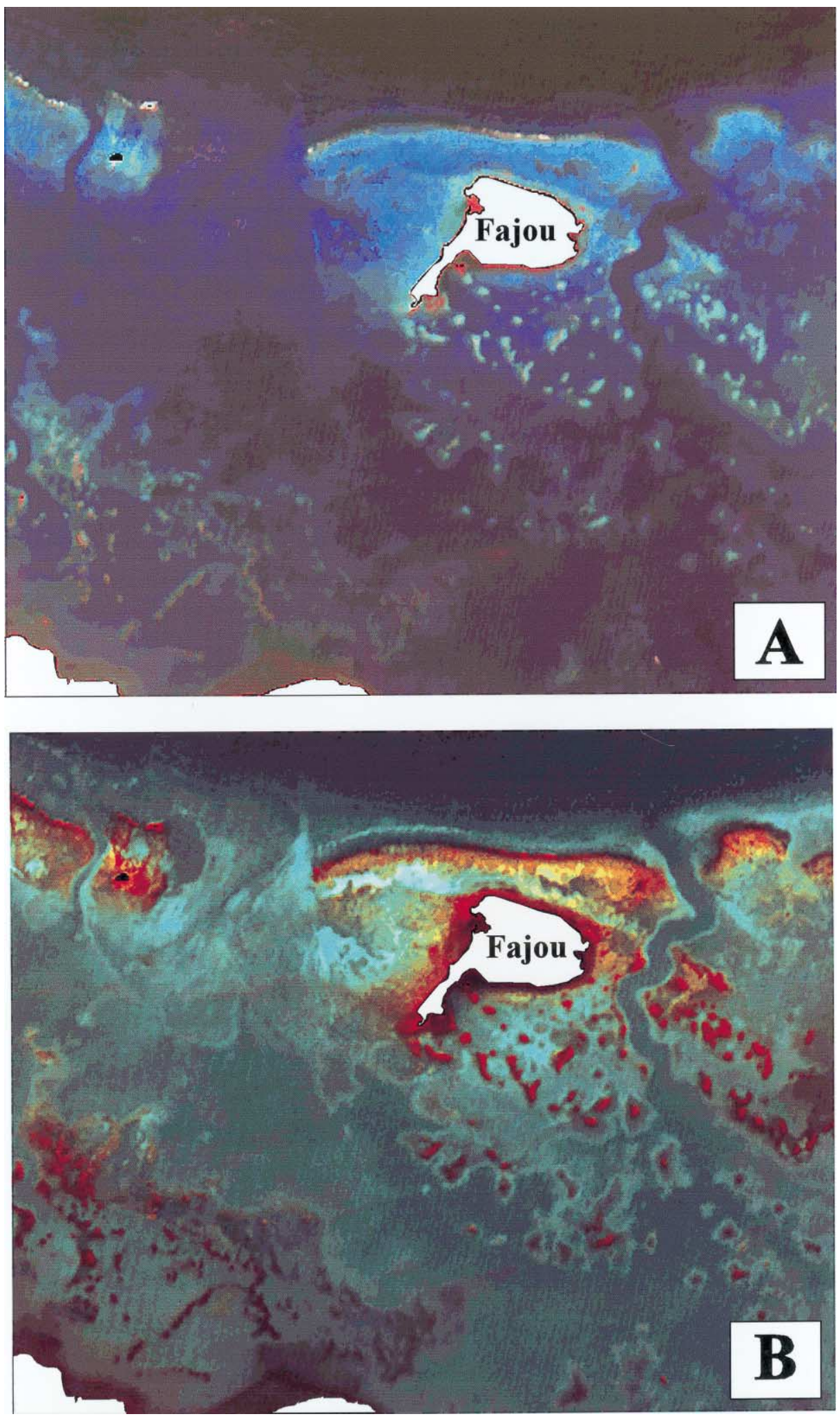

Figure 3. (A) Composition trichrome optimisée de la région de l'ilet Fajou en 1994 réalisée à partir des canaux XS1, XS2 et XS3 après masquages des parties terrestres et rehaussement du contraste. (B) Composition trichrome optimisée de la région de l'ilet Fajou réalisée à partir des canaux Can1, Can2 et Can3.

Figure 3. (A) Optimised trichromic composition of the ilet Fajou region realised with XS1, XS2 et XS3 channels after terrestrial areas were masked and contrast enhancement. (B) Optimised trichromic composition of the ilet Fajou region realised with Can1, Can2 et Can3 channels. 
de côté, la probabilité de ne pas être localisé à l'intérieur de la parcelle est très faible. Pour chacune des 250 parcelles, la profondeur, la nature du fond et les caractéristiques de la biocénose ont été observées en plongée sous-marine.

\subsection{Classifications supervisées}

Le principe général de la technique de classification supervisée est de comparer la signature spectrale de chaque pixel de l'image à classer à celle d'objets parfaitement connus (parcelles tests). Les pixels des «parcelles tests » servent à calculer une référence spectrale moyenne pour un thème donné. Un algorithme dit « du maximum de vraisemblance » (Fralit, 1981) attribue à chaque pixel un code correspondant à l'une des classes définies par les parcelles tests. À partir d'un seuil de rejet fixé par l'utilisateur (dans cette étude: $0,2 \%$ ), les pixels dont l'appartenance à une classe est statistiquement non significative sont éliminés (affectés à un code de rejet). Dans l'élaboration des parcelles, plus de 40 classes sont retenues. Il est nécessaire de grouper ensuite les thèmes correspondant à un même biotope, mais qui, situés à des profondeurs différentes, ne présentaient pas la même signature spectrale.

\subsection{Estimation de la fiabilité des classifications}

Dans le but d'estimer la validité de la carte thématique réalisée, 120 sites distribués aléatoirement sont retenus, localisés (GPS) et étudiés sur le terrain. La fiabilité de la carte est estimée, d'une part, en confrontant la thématique de la carte et celle de ces nouveaux sites et, d'autre part, en construisant une matrice de confusion. Pour élaborer cette matrice, de nouvelles surfaces de référence sont délimitées à partir des nouveaux relevés de terrain, lorsque ceux-ci sont associés à des structures homogènes et de dimensions suffisantes $\left(>3600 \mathrm{~m}^{2}\right)$. Pour chaque thème, l'ensemble des relevés doit rassembler au moins 50 pixels. En raison de l'intrication des biotopes dans le Grand Cul-de-Sac Marin, pour certains thèmes, il n'est pas possible d'identifier un nombre suffisant de parcelles répondant à ces critères; dans ce cas, les parcelles tests sont utilisées comme références.

\subsection{Traitement particulier des zones de mangrove}

Afin d'étendre la cartographie à l'ensemble des zones sous influence marine (niveau des plus hautes mers), les mangroves à palétuviers, ainsi que les zones marécageuses qui leur succèdent vers la côte, sont prises en considération. Ces biotopes médio- et supralittoraux étant à la limite du cadre de l'étude, une technique plus rapide est utilisée. Les zones immergées sont d'abord masquées par seuillage à l'aide du canal XS3. Une amélioration interactive du contraste est réalisée pour chaque canal, en privilégiant la mise en valeur des zones de palétuviers et de marais. Une classification non supervisée est ensuite élaborée sur la trichromie construite à partir de ces canaux. Avec l'assistance d'un phytosociologiste spécialiste des mangroves (D. Imbert, laboratoire de biologie végétale, université des Antilles et de la Guyane), une classification a été réalisée pour discriminer les principales formations végétales, puis regrouper certaines des classes ainsi construites.

Les principales étapes du traitement des données XS de SPOT sont résumées dans la figure 4.

\section{RÉSULTATS}

\section{1. Établissement de la carte biocénotique}

La carte thématique des peuplements marins du Grand Cul-de-Sac Marin de Guadeloupe est présentée figure 5. Les 32 thèmes retenus sont identifiés dans le tableau II. Les herbiers de phanérogames marines sont décrits en détail et représentés par 11 thèmes différents (code 2 à 12). Les communautés benthiques récifales font l'objet de 4 thèmes (code 16 à 19).

\subsection{Surfaces occupées par les différents thèmes}

La surface totale cartographiée est de 34057 ha. Cependant, certains codes correspondent à des fonds qui ne sont pas identifiables (classe de rejet : 1, eaux du large : 28 et zones turbides : 24). Une fois leur surface soustraite de la carte, une identité écologique est attribuée aux 23682 ha restants. Les mangroves et les marais en eau salée sont très intriqués avec la forêt marécageuse et les marais en 


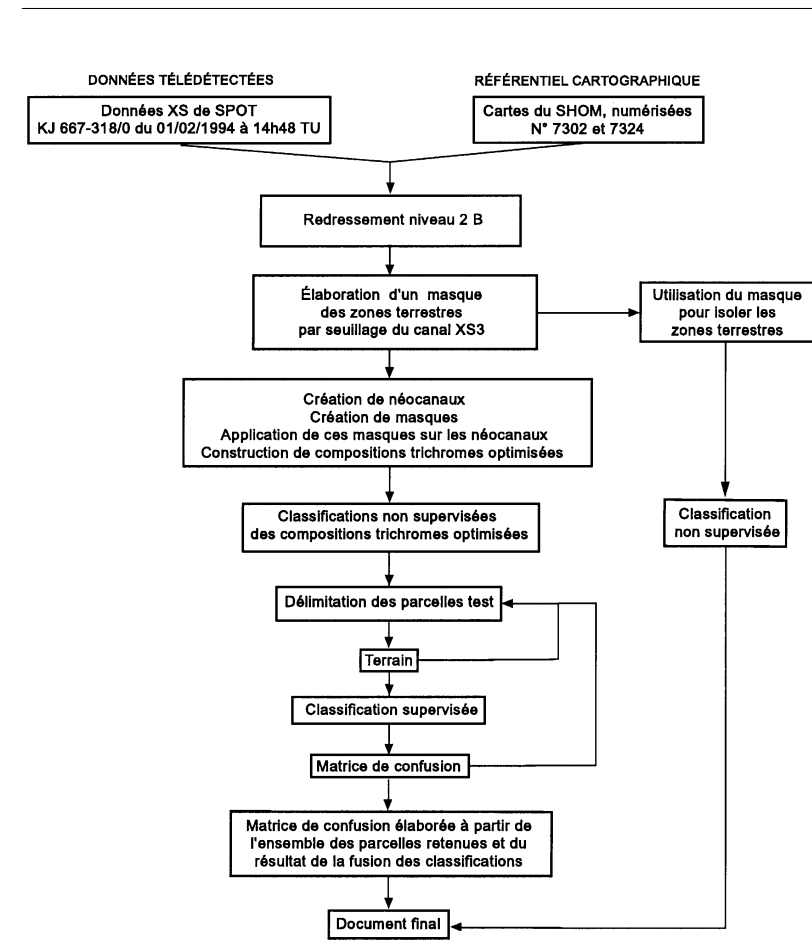

Figure 4. Principales étapes du traitement des données XS du satellite SPOT.

Figure 4. Diagram of the processing of XS SPOT data.

eau douce. Ce phénomène justifie la cartographie de ces biotopes terrestres. Forêts marécageuses et marais d'eau douce occupent 1489 ha. Les biotopes purement marins représentent 22193 ha.

Les zones de mangrove et les marais maritimes herbacés occupent 2692 ha, soit 12,1\% des biotopes marins. Les communautés infralittorales stricto sensu occupent 19500 ha. Elles sont réparties entre 15705 ha de fonds meubles et 3795 ha de fonds durs. Les substrats durs sont constitués de roches non récifales ( 2788 ha) et de formations coralliennes (1 $007 \mathrm{ha})$. Seuls 113 ha, soit $11,2 \%$ des zones récifales, sont occupés par des communautés coralliennes relativement florissantes (code 19) dont le taux de couverture par des coraux vivants dépasse $25 \%$. Ce travail cartographique met en évidence le faible taux de couverture corallienne et l'état de dégradation, déjà important en 1994, des récifs du Grand Cul-de-Sac Marin.

Différents types de fonds sédimentaires sont distingués par leur luminance particulière, qu'ils soient ou non occupés par des peuplements végétaux. Après une étude granulométrique réalisée sur le terrain, ces substrats meubles ont été subdivisés en 5614 ha de fonds de sable (incluant 103 ha de sédiments grossiers), 2863 ha de fonds sablo-vaseux et 5528 ha de fonds vaseux.

Les herbiers de phanérogames marines occupent une place prépondérante dans le Grand Cul-de-Sac Marin, 8220 ha de substrats meubles et représentent $42 \%$ des biotopes infralittoraux cartographiés. Ces herbiers sont essentiellement constitués par la phanérogame marine Thalassia testudinum (König), quelquefois associée à Syringodium filiforme (Kützing). Plusieurs catégories d'herbiers, correspondant à différents taux de couverture végétale pour un type de sédiment donné, ont été séparées. La possibilité de détailler les herbiers de phanérogames marines et les mesures in situ de la biomasse et de la production primaire des phanérogames (également effectuées en 1994) ont permis d'estimer la production annuelle en matière végétale des herbiers du Grand Cul-de-Sac Marin à 45800 t (p.s.) pour cette année (Chauvaud, 1997).

\subsection{Validité de la carte proposée}

Les relevés témoins effectués sur le terrain (au nombre de 120) permettent d'établir a posteriori l'adéquation entre la carte thématique et les observations : 111 relevés correspondent à la thématique proposée par la carte, et 9 seulement en diffèrent. Le risque de rencontrer sur le terrain une thématique différente de celle de la carte est de 7,5\%. Par ailleurs, dans 4 des 9 cas d'erreur, il y a confusion entre des thèmes concernant les herbiers. La probabilité de confusion entre des biocénoses différentes est donc plus faible $(4,2 \%)$. L'élaboration d'une matrice de confusion conforte ces résultats (tableau III). La fiabilité globale de la carte est de $95,7 \%$ et la confusion la plus forte est de $11 \%$ (entre les codes 17, massifs coralliens morts sur fond de sable, et 27 , fonds de vase profonds).

Dans la littérature concernant le milieu marin, très peu de documents issus de la télédétection sont associés à des critères de fiabilité. L'ultime vérification in situ de la carte produite et l'élaboration d'une matrice de confusion (Plourde et Thibault, 1985 ; Story et al., 1986) ont rarement été réalisées. Ces deux approches complémentaires sont utiles pour vérifier l'accord entre le document final et la réalité du terrain.

Pour réaliser une matrice de confusion fiable, des surfaces homogènes doivent être définies, de préférence non 


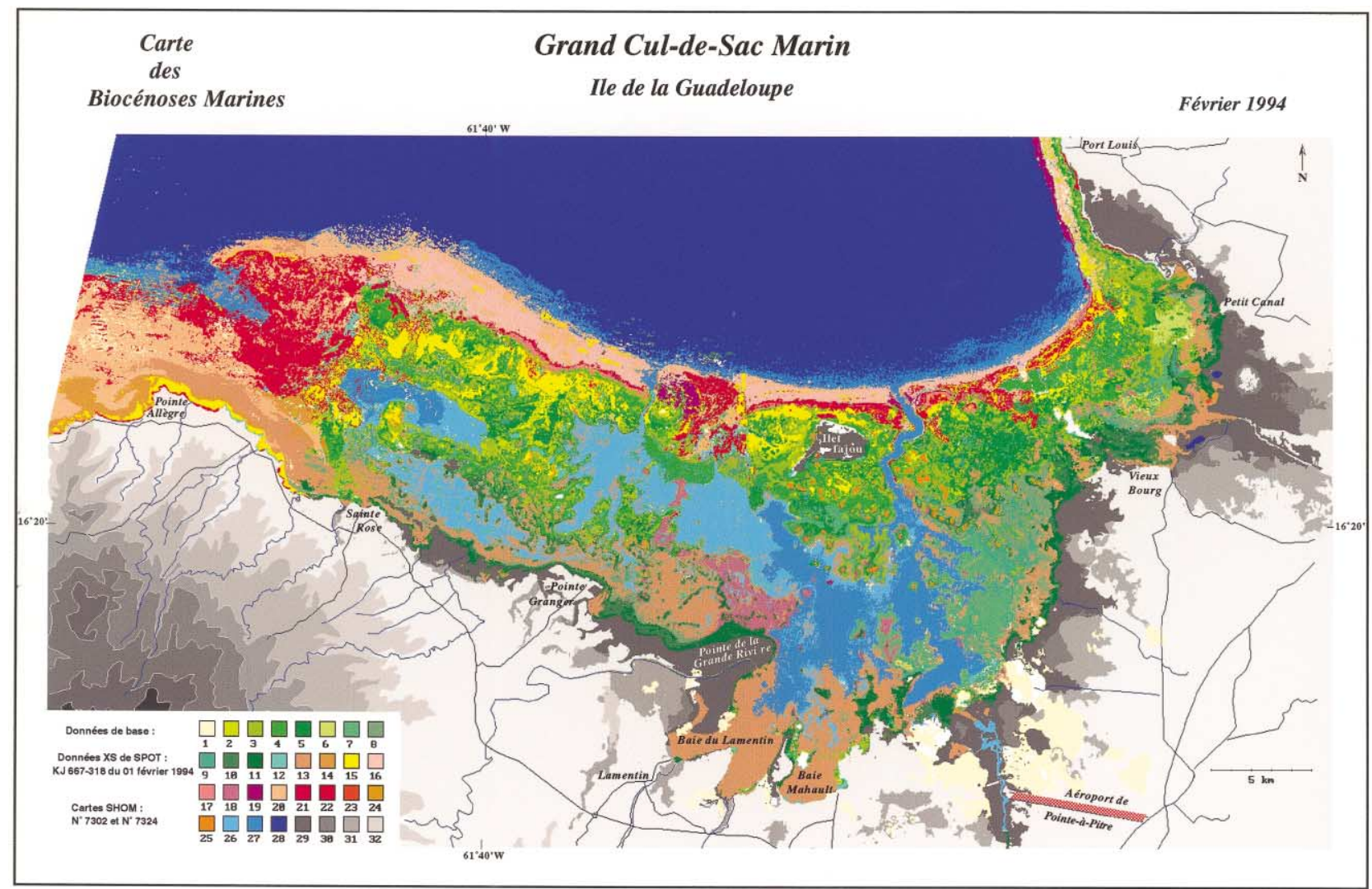

Figure 5. Carte thématique du Grand Cul-de-Sac Marin.

Figure 5. Thematic map of the Grand Cul-de-Sac Marin.

impliquées dans le traitement de l'image. Dans la présente étude, la fiabilité effective de la carte a été privilégiée par rapport à son estimation. Les 250 parcelles tests ont été utilisées pour l'élaboration des classifications, ce qui a permis d'y intégrer une très riche information de terrain. La conséquence de ce choix est qu'il n'a pas été possible d'élaborer de nouvelles références pour certains des thèmes cartographiés. Le fait que les parcelles tests aient partiellement contribué à l'élaboration de la matrice de confusion a conduit à minimiser la confusion réelle, mais la confrontation de la carte et des relevés ponctuels confirme, dans une large mesure, la bonne adéquation entre le document produit et la réalité du terrain.

\section{DISCUSSION ET CONCLUSIONS}

La cartographie des biocénoses marines côtières, en zones intertropicales, à partir de l'imagerie aérienne ou satellitaire, impose certaines contraintes aux données télédétectées : a) résolution physique adaptée au sujet de l'étude ; b) énergie lumineuse dans la gamme de longueurs d'ondes ayant un pouvoir élevé de pénétration dans l'eau, permettant ainsi de discriminer des objets profonds.

\subsection{Résolution}

La résolution des données télédétectées est un paramètre extrêmement important qui doit être en adéquation avec les dimensions des objets à cartographier. Plusieurs cartographies thématiques des milieux côtiers tropicaux ont été réalisées à partir des données MSS (Multi Spectral Scanner) du satellite LANDSAT. Ackleson et Klemas (1987) ne signalent pas de difficultés particulières liées à la résolution de ces données pour l'inventaire d'herbiers de phanérogames marines tropicales. En revanche, Pi- 
Tableau II. Thèmes identifiés dans le Grand Cul-de-Sac Marin, surfaces correspondantes sur la carte et importance de chaque thème.

Table II. Themes identified on the map of the Grand Cul-de-Sac Marin, corresponding surfaces and relative importance of each theme.

\begin{tabular}{|c|c|c|c|c|}
\hline $\begin{array}{l}\text { Code du } \\
\text { thème }\end{array}$ & Thème associé à chaque code & $\begin{array}{c}\text { Nombre } \\
\text { de } \\
\text { pixels }\end{array}$ & $\begin{array}{c}\text { Surface } \\
\text { cartographiée } \\
\text { (ha) }\end{array}$ & $\begin{array}{c}\text { Part de } \\
\text { chaque } \\
\text { thème } \\
(\%)\end{array}$ \\
\hline 1 & Indéterminé (nuages, classe de rejet) & 2437 & 97 & \\
\hline 2 & Herbiers à $T$. testudinum sur sable couverture végétale de 1 à $25 \%$ & 17441 & 698 & 2,9 \\
\hline 3 & Herbiers à $T$. testudinum sur sable couverture végétale de 26 à $50 \%$ & 35439 & 1418 & 6,0 \\
\hline 4 & Herbiers à T.testudinum sur sable couverture végétale de 51 à $75 \%$ & 34827 & 1393 & 5,9 \\
\hline 5 & Herbiers à T. testudinum sur sable couverture végétale de 76 à $100 \%$ & 21756 & 870 & 3,7 \\
\hline 6 & Herbiers à $T$. testudinum sur sable-vaseux couverture végétale de 1 à $25 \%$ & 2251 & 90 & $\mathbf{0 , 4}$ \\
\hline 7 & Herbiers à $T$. testudinum sur sable-vaseux couverture végétale de 26 à $50 \%$ & 16004 & 640 & 2,7 \\
\hline 8 & Herbiers à $T$. testudinum sur sable-vaseux couverture végétale de 51 à $75 \%$ & 17491 & 700 & 3,0 \\
\hline 9 & Herbiers à $T$. testudinum sur sable-vaseux couverture végétale de 76 à $100 \%$ & 17877 & 715 & $\mathbf{3 , 0}$ \\
\hline 10 & Herbiers à T. testudinum sur vase couverture végétale de 26 à $50 \%$ & 12385 & 495 & 2,1 \\
\hline 11 & Herbiers à $T$. testudinum sur vase couverture végétale de 76 à $100 \%$ & 17834 & 713 & 3,0 \\
\hline 12 & Herbiers à $T$. testudinum sur sédiment grossier hétérogène & 2578 & 103 & $\mathbf{0 , 4}$ \\
\hline 13 & Vase nue & 64435 & 2577 & 10,9 \\
\hline 14 & Sable-vaseux nu & 8229 & 329 & 1,4 \\
\hline 15 & Sable nu & 18720 & 749 & 3,2 \\
\hline 16 & Dalle à faible taux de recouvrement corallien & 18219 & 729 & 3,1 \\
\hline 17 & Massifs coralliens nécrosé sur fond de sable & 1579 & 63 & $\mathbf{0 , 3}$ \\
\hline 18 & Herbiers à Thalassia testudinum avec massifs coralliens vivants & 8255 & 330 & 1,4 \\
\hline 19 & Fonds coralliens & 2824 & 113 & $\mathbf{0 , 5}$ \\
\hline 20 & Roche nue & 36947 & 1478 & 6,2 \\
\hline 21 & Platier coralliens mort avec une forte couverture par les algues & 2544 & 102 & 0,4 \\
\hline 22 & Roche avec une forte couverture alguale & 32781 & 1311 & 5,5 \\
\hline 23 & Algues sur fonds sédimentaires & 142 & 6 & $\mathbf{0 , 0}$ \\
\hline 24 & Zone turbide & 434 & 17 & \\
\hline 25 & Herbiers affleurants avec coraux dispersés & 1352 & 54 & 0,2 \\
\hline 26 & Fonds de vase peu profonds & 40541 & 1622 & 6,8 \\
\hline 27 & Fonds de vase profonds & 55079 & 2203 & 9,3 \\
\hline 28 & Eaux du large & 256506 & 10260 & \\
\hline 29 & Mangrove & 58132 & 2325 & 9,8 \\
\hline 30 & Marais herbacés & 9175 & 367 & 1,5 \\
\hline 31 & Forêt humide & 35875 & 1435 & 6,1 \\
\hline 32 & Marais d'eau douce & 1327 & 53 & $\mathbf{0 , 2}$ \\
\hline \multicolumn{2}{|r|}{ Total } & 851416 & 34057 & \\
\hline
\end{tabular}

razzoli (1982) et Thomassin et al., (1985) notent que les dimensions du pixel des images MSS de LANDSAT $(79 \mathrm{~m} \times 79 \mathrm{~m})$ ne sont pas adaptées à la cartographie des zones récifales hétérogènes.

Aujourd'hui, les capteurs TM (Thematic Mapper) du satellite LANDSAT et XS de SPOT sont plus performants. Armstrong et al. (1985) et Armstrong (1993) soulignent que les images TM de LANDSAT $(30 \mathrm{~m} \times$ $30 \mathrm{~m}$ ) sont adaptées à la cartographie de récifs de très grandes tailles, mais pas à celle des milieux coralliens caraïbes de dimensions souvent réduites. Thomassin et al. (1985), Kuchler et al. (1986), Baimbridge et Reichelt (1988) et Chauvaud (1997) notent que par sa résolution en mode multispectral $(20 \mathrm{~m} \times 20 \mathrm{~m})$ SPOT est mieux adapté que LANDSAT à la cartographie de zones côtières tropicales de nature complexe. Ainsi, Bour et al. (1986) ont délimité l'habitat de mollusques d'intérêt commercial sur les récifs de Nouvelle-Calédonie à l'aide de données SPOT. D'autres cartographies de biocénoses marines côtières ont été réalisées à l'aide des données 
Tableau III. Matrice de confusion. (les valeurs $<1$ ne sont pas figurées).

Table III. Error matrix (the values $<1$ are not presented).

XS de SPOT (Belsher et al., 1990 ; Courboulès et al., 1987, 1988, 1992 ; Loubersac et Populus, 1986). Cependant, Courboulès et al. (1992) et Chauvaud (1997) signalent que les données SPOT, par leur résolution relativement faible, sont mal adaptées à certains milieux marins tropicaux, surtout lorsque l'intrication est importante et les variations de relief marquées. Dans ce cas, l'imagerie aérienne, d'une très haute finesse permet de s'affranchir des contraintes liées à la résolution (Bajjouk, 1996 ; Chauvaud, 1997, 1998 ; Courboulès, 1989 ; Manière et Jaubert, 1985 ; Manière et al., 1986, 1994). En comparant une image LANDSAT TM et des clichés aériens pris à la même période, Ramsey et Laine (1997) concluent également à la supériorité de l'imagerie aérienne sur les données satellitaires.

Dans le présent travail, les données SPOT se révèlent bien adaptées à la cartographie fine des biotopes marins du Grand Cul-de-Sac Marin. Ce résultat est lié à la structure particulière de la baie qui abrite des ensembles bionomiques relativement étendus et sur des fonds au relief modéré. La profondeur et le type de fond peuvent être considérés comme uniformes sur l'étendue d'un pixel, ce qui limite les risques de confusion lors de la classification. La résolution des données XS de SPOT permet donc de cartographier un secteur d'une richesse thématique importante.

\subsection{Pénétration bathymétrique et expression de la richesse thématique}

La télédétection en milieu marin est essentiellement limitée par l'atténuation des radiations lumineuses dans l'eau. Toutes les longueurs d'ondes n'ont pas le même pouvoir de pénétration (Jerlov, 1976). Que les images soient d'origine aérienne ou spatiale, la discrimination des objets profonds dépend de la sensibilité spectrale des émulsions photographiques ou des capteurs électroniques utilisés.

Pour les Antilles françaises, des scènes en provenance des satellites SPOT et LANDSAT sont disponibles. Les scènes LANDSAT TM couvrent une large bande spectrale, mais leur résolution relativement faible conduit à privilégier le satellite SPOT dont les trois canaux sont calés dans l'infrarouge, le rouge et le vert. L'absorption du proche infrarouge par les premiers centimètres d'eau 